\title{
Viscosity Measurements of Rocket Propellant RP-2 Over Wide Ranges of Temperature and Pressure
}

Hseen O. Baled ${ }^{1,}$, Robert M. Enick${ }^{1}$, Rajendar R. Mallepally ${ }^{2}$, Babatunde A. Bamgbade ${ }^{2}$, Mark A. McHugh², Matthew C. Billingsley ${ }^{3}$

${ }^{1}$ Department of Chemical and Petroleum Engineering, University of Pittsburgh, Pittsburgh, PA

${ }^{2}$ Department of Chemical and Life Science Engineering, Virginia Commonwealth University, Richmond, VA

${ }^{3}$ Air Force Research Laboratory (AFRL), Edwards Air Force Base, CA

* Corresponding author, e-mail: hob9@pitt.edu, phone: +1 (412) 3839032 and Fax: +1 (412) 6249639

Table S1. Detailed composition of rocket grade kerosene RP-2A and RP-2B used in this study, and RP-2 (POSF 12810) used in the work reported by Laesecke and Cousins ${ }^{3}$. Composition determined using two-dimensional gas chromatography (GCxGC) with simultaneous mass spectral (MS) and flame ionization detection (FID) as described in Reference 9

\begin{tabular}{|c|c|c|c|c|c|c|}
\hline \multirow[b]{2}{*}{ Hydrocarbon Type } & \multicolumn{2}{|c|}{ RP-2A (POSF 12427) } & \multicolumn{2}{|c|}{ RP-2B (POSF 13205) } & \multicolumn{2}{|c|}{ RP-2 (POSF 12810) } \\
\hline & Weight \% & Volume \% & Weight $\%$ & Volume \% & Weight $\%$ & Volume \% \\
\hline \multicolumn{7}{|l|}{ Aromatics } \\
\hline \multicolumn{7}{|l|}{ Alkylbenzenes } \\
\hline benzene (C06) & $<0.01$ & $<0.01$ & $<0.01$ & $<0.01$ & $<0.01$ & $<0.01$ \\
\hline toluene $(\mathrm{C} 07)$ & $<0.01$ & $<0.01$ & $<0.01$ & $<0.01$ & $<0.01$ & $<0.01$ \\
\hline C2-benzene (C08) & $<0.01$ & $<0.01$ & $<0.01$ & $<0.01$ & $<0.01$ & $<0.01$ \\
\hline C3-benzene (C09) & $<0.01$ & $<0.01$ & $<0.01$ & $<0.01$ & $<0.01$ & $<0.01$ \\
\hline C4-benzene (C10) & 0.05 & 0.05 & $<0.01$ & $<0.01$ & 0.04 & 0.03 \\
\hline
\end{tabular}




\begin{tabular}{|c|c|c|c|c|c|c|}
\hline C5-benzene (C11) & 0.06 & 0.05 & $<0.01$ & $<0.01$ & 0.03 & 0.03 \\
\hline C6-benzene (C12) & $<0.01$ & $<0.01$ & 0.03 & 0.03 & $<0.01$ & $<0.01$ \\
\hline C7-benzene (C13) & 0.04 & 0.04 & 0.04 & 0.04 & 0.04 & 0.04 \\
\hline C8-benzene (C14) & 0.04 & 0.04 & $<0.01$ & $<0.01$ & $<0.01$ & $<0.01$ \\
\hline C9+-benzene $(\mathrm{C} 15+)$ & 0.08 & 0.07 & 0.03 & 0.02 & 0.03 & 0.03 \\
\hline Total Alkylbenzenes & 0.28 & 0.26 & 0.11 & 0.10 & 0.16 & 0.15 \\
\hline \multicolumn{7}{|c|}{ Diaromatics (Naphthalenes, Biphenyl, etc.) } \\
\hline diaromatic-C10 & $<0.01$ & $<0.01$ & $<0.01$ & $<0.01$ & $<0.01$ & $<0.01$ \\
\hline diaromatic-C11 & $<0.01$ & $<0.01$ & $<0.01$ & $<0.01$ & $<0.01$ & $<0.01$ \\
\hline diaromatic-C12 & 0.01 & $<0.01$ & $<0.01$ & $<0.01$ & $<0.01$ & $<0.01$ \\
\hline diaromatic-C13 & $<0.01$ & $<0.01$ & $<0.01$ & $<0.01$ & $<0.01$ & $<0.01$ \\
\hline diaromatic-C14+ & $<0.01$ & $<0.01$ & $<0.01$ & $<0.01$ & $<0.01$ & $<0.01$ \\
\hline Total Alkylnaphthalenes & 0.02 & 0.02 & $<0.01$ & $<0.01$ & $<0.01$ & $<0.01$ \\
\hline \multicolumn{7}{|c|}{ Cycloaromatics (Indans, Tetralins, etc.) } \\
\hline cycloaromatic-C09 & $<0.01$ & $<0.01$ & $<0.01$ & $<0.01$ & $<0.01$ & $<0.01$ \\
\hline cycloaromatic-C10 & 0.02 & 0.01 & $<0.01$ & $<0.01$ & $<0.01$ & $<0.01$ \\
\hline cycloaromatic-C11 & 0.03 & 0.03 & $<0.01$ & $<0.01$ & 0.01 & 0.01 \\
\hline cycloaromatic-C12 & 0.04 & 0.04 & 0.06 & 0.05 & 0.07 & 0.06 \\
\hline cycloaromatic-C13 & 0.16 & 0.14 & 0.10 & 0.09 & 0.09 & 0.08 \\
\hline cycloaromatic-C14 & 0.16 & 0.14 & 0.09 & 0.08 & 0.06 & 0.05 \\
\hline cycloaromatics-C15+ & 0.08 & 0.07 & 0.03 & 0.02 & 0.02 & 0.02 \\
\hline Total Cycloaromatics & 0.49 & 0.42 & 0.28 & 0.25 & 0.26 & 0.23 \\
\hline otal Aromatics & 0.80 & 0.70 & 0.39 & 0.35 & 0.42 & 0.38 \\
\hline
\end{tabular}

\section{Paraffins}

iso-Paraffins 


\begin{tabular}{|c|c|c|c|c|c|c|}
\hline C07 and lower-iso & 0.04 & 0.05 & $<0.01$ & $<0.01$ & 0.03 & 0.04 \\
\hline C08-isoparaffins & $<0.01$ & $<0.01$ & $<0.01$ & $<0.01$ & $<0.01$ & $<0.01$ \\
\hline C09-isoparaffins & $<0.01$ & $<0.01$ & 0.02 & 0.02 & $<0.01$ & $<0.01$ \\
\hline C10-isoparaffins & 0.11 & 0.12 & 2.09 & 2.30 & 1.18 & 1.30 \\
\hline C11-isoparaffins & 5.65 & 6.02 & 5.52 & 5.96 & 7.36 & 7.91 \\
\hline C12-isoparaffins & 9.54 & 10.19 & 6.38 & 6.91 & 8.24 & 8.87 \\
\hline C13-isoparaffins & 4.98 & 5.20 & 6.97 & 7.38 & 7.74 & 8.15 \\
\hline C14-isoparaffins & 4.29 & 4.45 & 6.65 & 6.99 & 6.66 & 6.96 \\
\hline C15-isoparaffins & 9.36 & 9.64 & 4.78 & 4.99 & 3.59 & 3.73 \\
\hline C16-isoparaffins & 2.42 & 2.48 & 1.00 & 1.04 & 1.18 & 1.22 \\
\hline C17-isoparaffins & 0.57 & 0.58 & 0.25 & 0.26 & 0.42 & 0.43 \\
\hline C18-isoparaffins & 0.18 & 0.19 & 0.03 & 0.03 & 0.15 & 0.15 \\
\hline C19-isoparaffins & 0.03 & 0.03 & 0.06 & 0.06 & 0.05 & 0.05 \\
\hline C20-isoparaffins & $<0.01$ & $<0.01$ & $<0.01$ & $<0.01$ & 0.01 & 0.01 \\
\hline C21-isoparaffins & $<0.01$ & $<0.01$ & $<0.01$ & $<0.01$ & $<0.01$ & $<0.01$ \\
\hline C22-isoparaffins & $<0.01$ & $<0.01$ & $<0.01$ & $<0.01$ & $<0.01$ & $<0.01$ \\
\hline C23-isoparaffins & $<0.01$ & $<0.01$ & $<0.01$ & $<0.01$ & $<0.01$ & $<0.01$ \\
\hline C24-isoparaffins & $<0.01$ & $<0.01$ & $<0.01$ & $<0.01$ & $<0.01$ & $<0.01$ \\
\hline Total iso-Paraffins & 37.18 & 38.95 & 33.75 & 35.94 & 36.63 & 38.82 \\
\hline \multicolumn{7}{|l|}{ n-Paraffins } \\
\hline $\mathrm{n}-\mathrm{C} 07$ & $<0.01$ & $<0.01$ & $<0.01$ & $<0.01$ & $<0.01$ & $<0.01$ \\
\hline $\mathrm{n}-\mathrm{C} 08$ & $<0.01$ & $<0.01$ & $<0.01$ & $<0.01$ & $<0.01$ & $<0.01$ \\
\hline $\mathrm{n}-\mathrm{C} 09$ & 0.01 & 0.01 & 0.04 & 0.05 & $<0.01$ & $<0.01$ \\
\hline $\mathrm{n}-\mathrm{C} 10$ & 0.42 & 0.45 & 0.08 & 0.09 & 0.03 & 0.03 \\
\hline $\mathrm{n}-\mathrm{C} 11$ & 5.84 & 6.24 & 0.08 & 0.09 & 0.07 & 0.08 \\
\hline $\mathrm{n}-\mathrm{C} 12$ & 4.46 & 4.70 & 0.08 & 0.09 & 0.14 & 0.15 \\
\hline $\mathrm{n}-\mathrm{C} 13$ & 0.50 & 0.52 & 0.03 & 0.03 & 0.15 & 0.16 \\
\hline $\mathrm{n}-\mathrm{C} 14$ & 0.05 & 0.05 & 0.02 & 0.02 & 0.14 & 0.15 \\
\hline
\end{tabular}




\begin{tabular}{|c|c|c|c|c|c|c|}
\hline $\mathrm{n}-\mathrm{C} 15$ & 0.01 & 0.01 & 0.01 & 0.01 & 0.08 & 0.08 \\
\hline $\mathrm{n}-\mathrm{C} 16$ & $<0.01$ & $<0.01$ & $<0.01$ & $<0.01$ & 0.04 & 0.04 \\
\hline $\mathrm{n}-\mathrm{C} 17$ & $<0.01$ & $<0.01$ & $<0.01$ & $<0.01$ & 0.02 & 0.02 \\
\hline $\mathrm{n}-\mathrm{C} 18$ & $<0.01$ & $<0.01$ & $<0.01$ & $<0.01$ & 0.01 & 0.01 \\
\hline $\mathrm{n}-\mathrm{C} 19$ & $<0.01$ & $<0.01$ & $<0.01$ & $<0.01$ & $<0.01$ & $<0.01$ \\
\hline $\mathrm{n}-\mathrm{C} 20$ & $<0.01$ & $<0.01$ & $<0.01$ & $<0.01$ & $<0.01$ & $<0.01$ \\
\hline $\mathrm{n}-\mathrm{C} 21$ & $<0.01$ & $<0.01$ & $<0.01$ & $<0.01$ & $<0.01$ & $<0.01$ \\
\hline $\mathrm{n}-\mathrm{C} 22$ & $<0.01$ & $<0.01$ & $<0.01$ & $<0.01$ & $<0.01$ & $<0.01$ \\
\hline $\mathrm{n}-\mathrm{C} 23$ & $<0.01$ & $<0.01$ & $<0.01$ & $<0.01$ & $<0.01$ & $<0.01$ \\
\hline Total n-Paraffins & 11.29 & 12.00 & 0.35 & 0.38 & 0.69 & 0.73 \\
\hline Total Paraffins (iso- + n-) & 48.47 & 50.95 & 34.1 & 36.32 & 37.32 & 39.55 \\
\hline \multicolumn{7}{|l|}{ Cycloparaffins } \\
\hline \multicolumn{7}{|l|}{ Monocycloparaffins } \\
\hline C1-monocyclo (C07) & $<0.01$ & $<0.01$ & $<0.01$ & $<0.01$ & $<0.01$ & $<0.01$ \\
\hline C2-monocyclo (C08) & $<0.01$ & $<0.01$ & 0.15 & 0.16 & 0.08 & 0.08 \\
\hline C3-monocyclo (C09) & 0.01 & 0.01 & 2.08 & 2.10 & 1.36 & 1.37 \\
\hline C4-monocyclo (C10) & 0.97 & 0.94 & 6.53 & 6.39 & 7.64 & 7.44 \\
\hline C5-monocyclo (C11) & 5.69 & 5.65 & 8.30 & 8.35 & 10.36 & 10.36 \\
\hline C6-monocyclo (C12) & 3.56 & 3.51 & 7.10 & 7.10 & 8.23 & 8.19 \\
\hline C7-monocyclo (C13) & 3.88 & 3.78 & 6.75 & 6.68 & 6.43 & 6.33 \\
\hline C8-monocyclo (C14) & 7.10 & 6.95 & 4.75 & 4.72 & 3.90 & 3.85 \\
\hline C9-monocyclo (C15) & 4.82 & 4.70 & 1.91 & 1.89 & 1.27 & 1.25 \\
\hline C10-monocyclo (C16) & 0.97 & 0.95 & 0.54 & 0.53 & 0.35 & 0.35 \\
\hline C11-monocyclo (C17) & 0.21 & 0.20 & 0.07 & 0.07 & 0.12 & 0.12 \\
\hline C12-monocyclo (C18) & 0.03 & 0.03 & 0.01 & 0.01 & 0.03 & 0.03 \\
\hline C13+-monocyclo (C19+) & $<0.01$ & $<0.01$ & $<0.01$ & $<0.01$ & 0.01 & 0.01 \\
\hline
\end{tabular}




\begin{tabular}{|c|c|c|c|c|c|c|}
\hline Total Monocycloparaffins & 27.25 & 26.72 & 38.19 & 37.99 & 39.80 & 39.38 \\
\hline \multicolumn{7}{|c|}{ Dicycloparaffins (Decalins, Bihexyls, etc.) } \\
\hline C08-dicycloparaffins & $<0.01$ & $<0.01$ & $<0.01$ & $<0.01$ & $<0.01$ & $<0.01$ \\
\hline C09-dicycloparaffins & $<0.01$ & $<0.01$ & 0.38 & 0.34 & 0.17 & 0.15 \\
\hline C10-dicycloparaffins & 1.04 & 0.92 & 2.24 & 2.01 & 2.57 & 2.28 \\
\hline C11-dicycloparaffins & 2.73 & 2.53 & 5.76 & 5.41 & 4.72 & 4.41 \\
\hline C12-dicycloparaffins & 2.74 & 2.56 & 6.02 & 5.69 & 5.32 & 5.00 \\
\hline C13-dicycloparaffins & 5.81 & 5.41 & 5.78 & 5.45 & 4.75 & 4.46 \\
\hline C14-dicycloparaffins & 4.69 & 4.37 & 2.28 & 2.15 & 1.41 & 1.32 \\
\hline C15-dicycloparaffins & 4.76 & 4.43 & 2.03 & 1.91 & 1.28 & 1.20 \\
\hline C16-dicycloparaffins & 0.10 & 0.09 & 0.04 & 0.04 & 0.03 & 0.02 \\
\hline C17+-dicycloparaffins & 0.03 & 0.03 & 0.01 & 0.01 & 0.02 & 0.02 \\
\hline Total Dicycloparaffins & 21.92 & 20.35 & 24.55 & 23.03 & 20.26 & 18.86 \\
\hline \multicolumn{7}{|l|}{ Tricycloparaffins } \\
\hline C10-tricycloparaffins & 0.06 & 0.04 & 0.10 & 0.08 & 0.09 & 0.08 \\
\hline C11-tricycloparaffins & 0.07 & 0.06 & 0.43 & 0.35 & 0.40 & 0.33 \\
\hline C12-tricycloparaffins & 0.46 & 0.38 & 0.99 & 0.83 & 1.06 & 0.88 \\
\hline C13-tricycloparaffins & 0.71 & 0.59 & 1.15 & 0.96 & 0.56 & 0.47 \\
\hline C14-tricycloparaffins & 0.19 & 0.15 & 0.07 & 0.06 & 0.04 & 0.04 \\
\hline C15-tricycloparaffins & 0.03 & 0.02 & 0.01 & 0.01 & $<0.01$ & $<0.01$ \\
\hline C16-tricycloparaffins & 0.03 & 0.02 & $<0.01$ & $<0.01$ & 0.02 & 0.02 \\
\hline C17-tricycloparaffins & $<0.01$ & $<0.01$ & $<0.01$ & $<0.01$ & $<0.01$ & $<0.01$ \\
\hline Total Tricycloparaffins & 1.55 & 1.28 & 2.77 & 2.31 & 2.20 & 1.82 \\
\hline Total Cycloparaffins & 50.72 & 48.36 & 65.51 & 63.33 & 62.25 & 60.07 \\
\hline
\end{tabular}




\section{CALIBRATION RESULTS}

The calibration results of the rolling-ball viscometer are shown in Figure S1. The calibration constant values, $k$, are presented as a function of pressure for each isotherm.

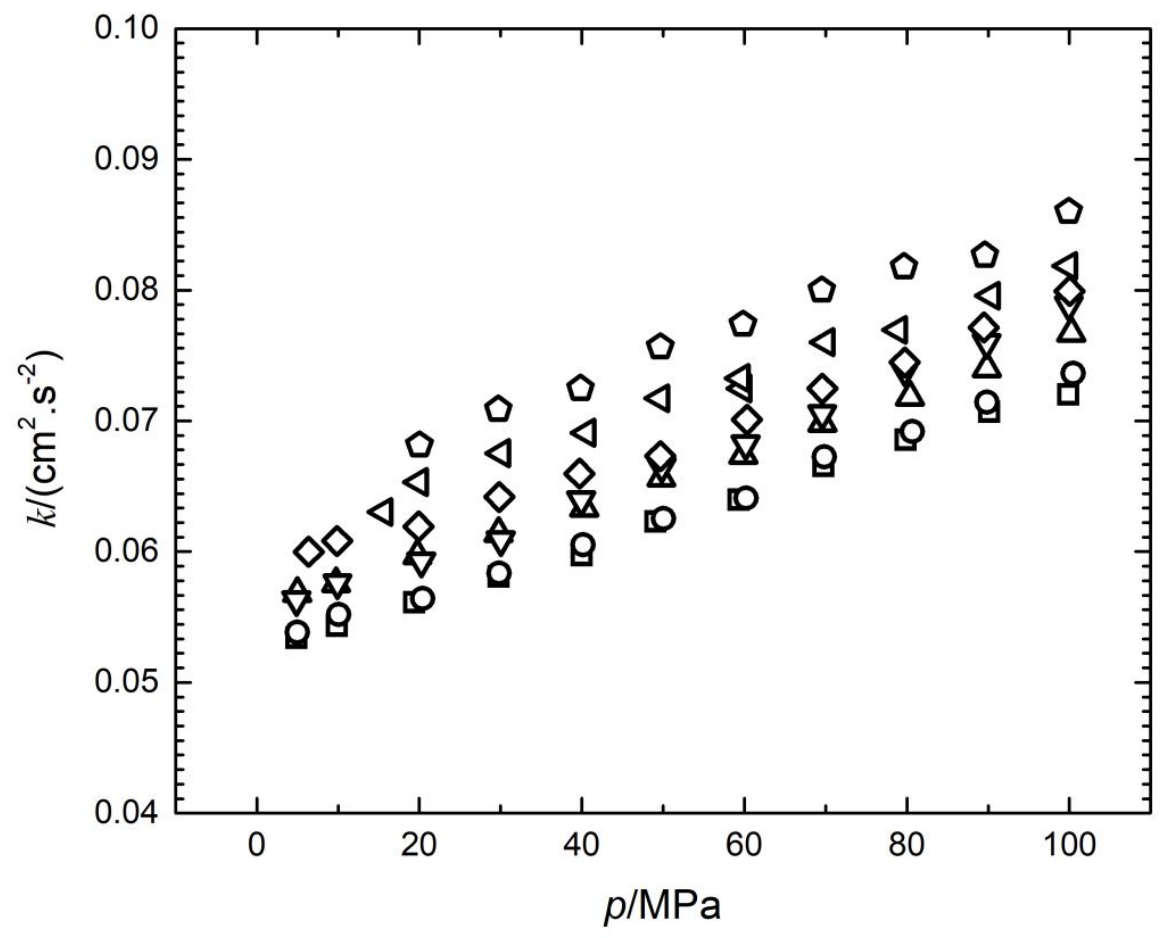

Figure S1. Rolling-ball viscometer calibration constant, $k$, at different pressures, $p$, measured with n-dodecane at a tilt angle of $7^{\circ}: \square 298 \mathrm{~K}, \bigcirc 324 \mathrm{~K}, \triangle 373 \mathrm{~K}, \nabla 423 \mathrm{~K}, \diamond 473 \mathrm{~K}, \triangleleft 524$ $\mathrm{K}, \mathrm{Q} 573 \mathrm{~K}$. 